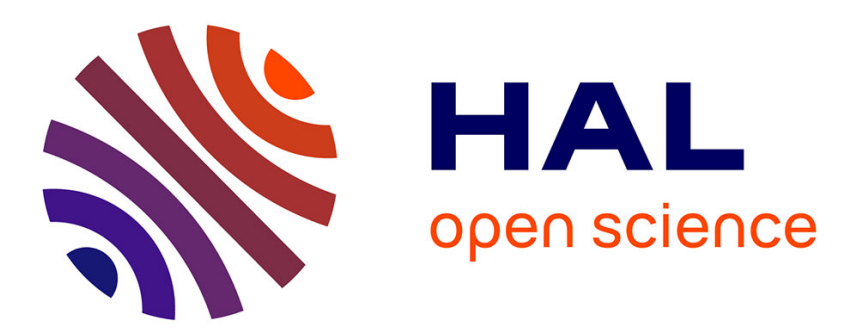

\title{
Le transistor-thyristor métal-oxyde-semiconducteur (T2 MOS)
}

Henri Tranduc, P. Rossel, Mohamed Gharbi, Jean-Louis Sanchez, G. Charitat

\section{To cite this version:}

Henri Tranduc, P. Rossel, Mohamed Gharbi, Jean-Louis Sanchez, G. Charitat. Le transistor-thyristor métal-oxyde-semiconducteur (T2 MOS). Revue de Physique Appliquée, 1985, 20 (8), pp.575-581. 10.1051/rphysap:01985002008057500 . jpa-00245372

\section{HAL Id: jpa-00245372 https://hal.science/jpa-00245372}

Submitted on 1 Jan 1985

HAL is a multi-disciplinary open access archive for the deposit and dissemination of scientific research documents, whether they are published or not. The documents may come from teaching and research institutions in France or abroad, or from public or private research centers.
L'archive ouverte pluridisciplinaire HAL, est destinée au dépôt et à la diffusion de documents scientifiques de niveau recherche, publiés ou non, émanant des établissements d'enseignement et de recherche français ou étrangers, des laboratoires publics ou privés. 


\title{
Le transistor-thyristor métal-oxyde-semiconducteur (T2 MOS) $\left(^{*}\right)$
}

\author{
H. Tranduc, P. Rossel, M. Gharbi, J. L. Sanchez et G. Charitat \\ Laboratoire d'Automatique et d'Analyse des Systèmes du Centre National de la Recherche Scientifique, \\ 7, avenue du Colonel Roche, 31077 Toulouse Cedex, France
}

(Reçu le 12 mars 1985, révisé le 22 avril, accepté le 6 mai 1985)

\begin{abstract}
Résumé. - On présente un nouveau composant planar contrôlé par un effet " Métal-Oxyde-Semiconducteur ", dont les caractéristiques électriques peuvent être : i) de type transistor MOS, ii) de type thyristor MOS ou bien iii) être la combinaison des deux précédentes. On l'appelle le « transistor-thyristor MOS » ou $\mathrm{T}^{2} \mathrm{MOS}$. Un exemple de réalisation technologique est décrit et les caractéristiques électriques sont présentées. Des variantes de configurations par rapport à la géométrie de ce prototype sont suggérées. Des utilisations possibles de ces structures sont évoquées.
\end{abstract}

\begin{abstract}
A new MOS gate-controlled planar device is proposed. It's electrical characteristics could be either : i) MOS transistor like, ii) MOS thyristor like or iii) a combination of the two previous devices. This device is called the "MOS transistor-thyristor " $\left(\mathrm{T}^{2} \mathrm{MOS}\right)$. The electrical characteristics measured on a small-size prototype are presented. Different geometrical and technological configurations for this kind of device are also suggested and the various areas for possible applications are discussed.
\end{abstract}

L'une des directions d'évolution des composants de l'électronique de puissance est celle de l'intégration monolithique d'un circuit de commande, logique et (ou) analogique, en technologie bipolaire et (ou) MOS, et d'un ou de plusieurs éléments de sortie qui constituent les interrupteurs de puissance [1-3]. Ces derniers sont, soit des transistors bipolaires [4], soit des transistors MOS (T MOS) [5], soit des thyristors MOS (Thy MOS) [6]. Les éléments de commande et de sortie sont réalisés à ce jour en technologie planar et sont compatibles avec les technologies des filières bipolaires ou (et) MOS.

Nous proposons une nouvelle configuration de structure de puissance compatible avec ces deux types de technologies et qui présente : i) soit une caractéristique de sortie de type T MOS, ii) soit une caractéristique de sortie de type Thy MOS, iii) soit une caractéristique qui est la superposition des deux précédentes, et qui sera alors appelée caractéristique de type $\mathrm{T}^{2}$ MOS. Nous décrirons la structure d'un prototype de.ce nouveau composant puis présenterons ses caractéristiques électriques. Des variantes de configuration seront proposées; quelques applications seront suggérées.

$\left({ }^{*}\right)$ Travail partiellement soutenu sur contrat DIELI.

\section{Structure du composant.}

Nous décrirons tout d'abord la structure la plus simple pour ce dispositif $\mathrm{T}^{2} \mathrm{MOS}$ : les autres configurations feront l'objet d'une partie du paragraphe 3. Ce composant (Fig. 1) présente horizontalement une structure similaire à celle d'un transistor MOS double-diffusé (D MOS) latéral [7]. Une source $\mathrm{N}^{+}$, une zone de canal $\mathrm{P}$, recouverte par un oxyde mince et par une grille, et un drain $\mathrm{N}^{+}$sont réalisés par les processus de diffusion ou de double-diffusion standardisés. Les trois électrodes de source(S), drain (D) et grille (G) sont coplanaires. Ce transistor D MOS est réalisé dans une couche $\mathrm{N}^{-}$épitaxiée sur un substrat $\mathrm{P}^{+}$. Un contact pris sur la face arrière de ce substrat constitue une nouvelle électrode appelée anode. Une configuration de thyristor vertical [8] est ainsi obtenue dans la structure multicouche constituée par le substrat $\mathrm{P}^{+}$, la couche épitaxiée $\mathrm{N}^{-}$, la diffusion $\mathbf{P}$ et la diffusion $\mathrm{N}^{+}$. Le déclenchement de ce thyristor est assuré par la mise en conduction du transistor MOS, situé entre les deux zones $\mathbf{N}$, lorsqu'une couche inversée est générée au droit de l'interface silicium P-silice, quand la grille est rendue positive par rapport à la source. Cette structure est réalisée sous forme de bandes interdigitées et la figure 1.1 précise la coupe d'une partie de la zone active. Le court-circuit entre régions 

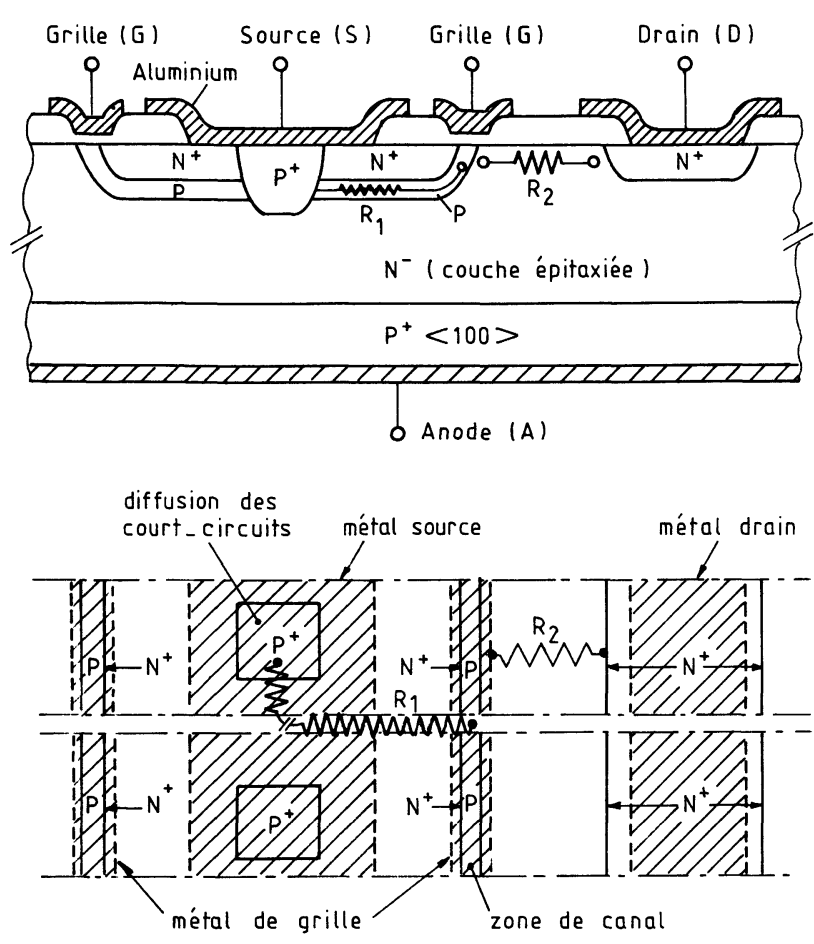

1.1

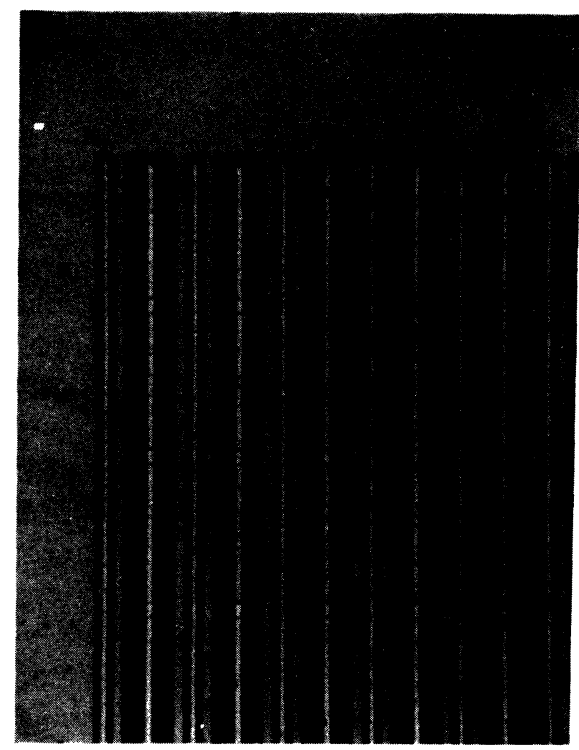

1.2

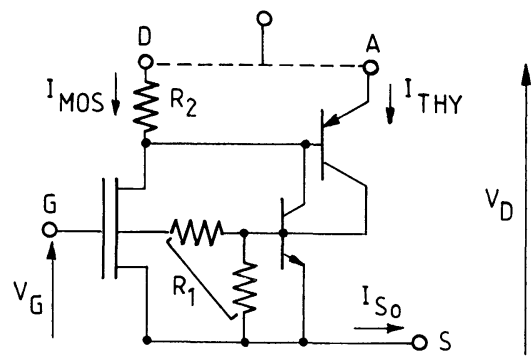

1.3
$\mathrm{N}$ et $\mathrm{P}$ - nécessaire pour référencier le potentiel de la zone $\mathrm{P}$ en fonctionnement $\mathrm{T}$ MOS - est assuré par de petites cellules diffusées $\mathrm{P}^{+}$, de forme carrée, uniformément réparties tout au long du doigt constitué par la double diffusion $\mathrm{N}^{+}, \mathrm{P}$ (Figs. 1.1, 1.2). La distance entre ces cellules $\mathrm{P}^{+}$est déterminée en vue de favoriser le déclenchement en mode thyristor : ces courts-circuits localisés entre zone $\mathrm{N}^{+}$et $\mathrm{P}$ via la diffusion $\mathrm{P}^{+}$jouent alors le rôle de courts-circuits de cathode; leur interdistance impose la valeur de la résistance $R_{1}$ qui apparaît sur le schéma équivalent de la figure 1.3 , valeur qui régit comme l'a montré Hachad [9] le mode de déclenchement en thyristor.

Les prototypes que nous avons réalisés à ce jour et dont nous présenterons les caractéristiques sont prévus pour fonctionner jusqu'à une centaine de volts environ, et conduire un courant de l'ordre de l'ampère : ils sont essentiellement destinés à prouver la faisabilité de la fonction électrique schématisée sur la figure 1.4 qui représente le schéma équivalent du composant. Les caractéristiques technologiques sont les suivantes : largeur de source de l'ordre de $40 \mu \mathrm{m}$ par doigt, longueur du canal de $5 \mu \mathrm{m}$ environ, distance source-drain de $25 \mu \mathrm{m}$ et épaisseur d'épitaxie $(\rho=20 \Omega \mathrm{cm})$ de $25 \mu \mathrm{m}$, distance transversale entre courts-circuits $\mathrm{P}^{+}$ de $100 \mu \mathrm{m}$, épaisseur d'oxyde mince de $1500 \AA$, épaisseur d'oxyde épais de $8000 \AA$, largeur de canal du D MOS de $24 \mathrm{~mm}$. La taille des puces est de l'ordre du millimètre carré.

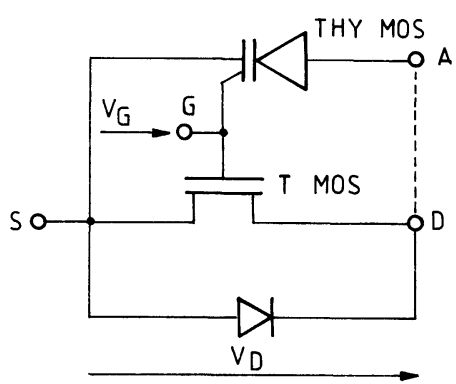

1.4

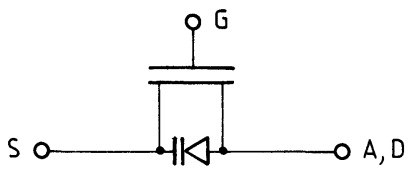

1.5

Fig. 1. - Le dispositif «Transistor-Thyristor MOS » $\mathrm{T}^{2}$ MOS. 1.1) Coupe et vue du dessus. 1.2) Photographie d'une partie du composant. 1.3) Schéma électrique équivalent localisé. 1.4) Les fonctions électriques réalisées. 1.5) Symbole représentatif.

[The MOS Transistor-Thyristor $\mathrm{T}^{2}$ MOS. 1.1) Cross-section and top-view. 1.2) Partial view of the device. 1.3) Equivalent electrical circuit. 1.4) Electronic functions performed. 1.5) $\mathrm{T}^{2}$ MOS graphic symbol.] 


\section{Fonctionnement du composant.}

Le $\mathrm{T}^{2}$ MOS est constitué par deux composants : un transistor MOS et un thyristor MOS qui peuvent être utilisés séparément ou mis en parallèle par métallisation interne ou par microsoudure ou par connexion externe au boîtier, pour réaliser des fonctions élec-
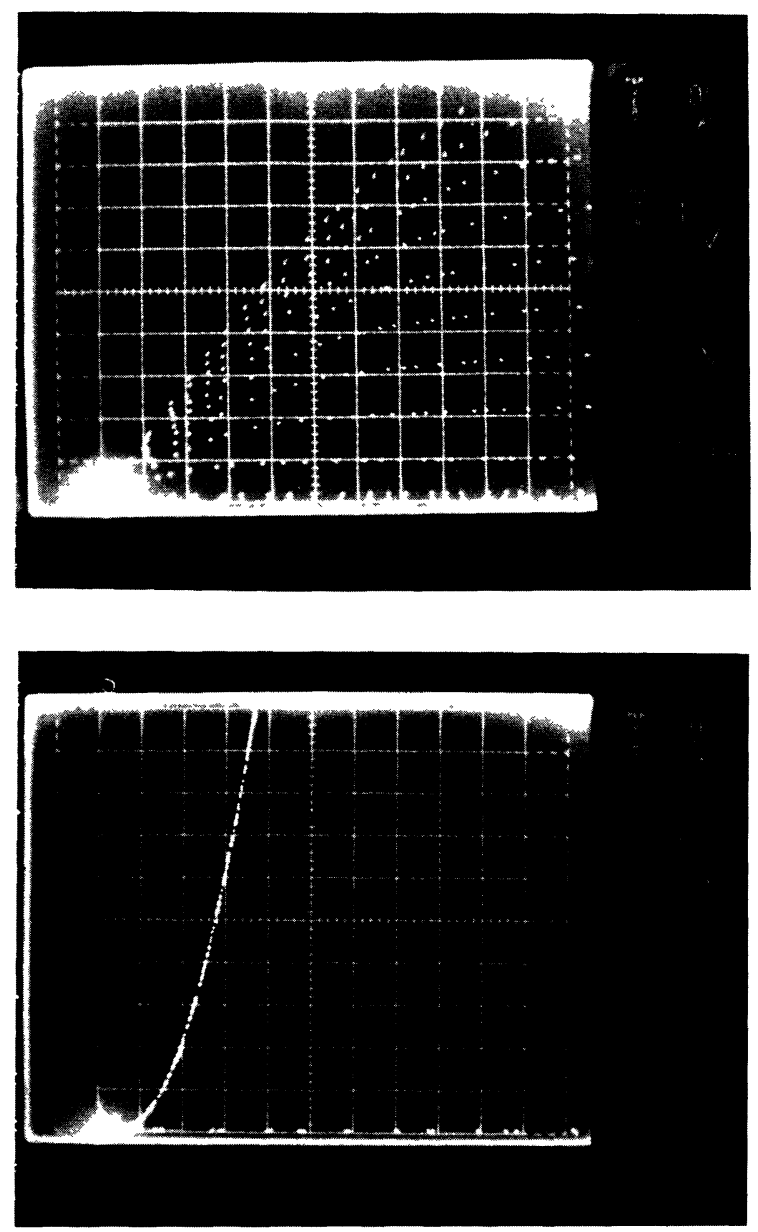

(b)
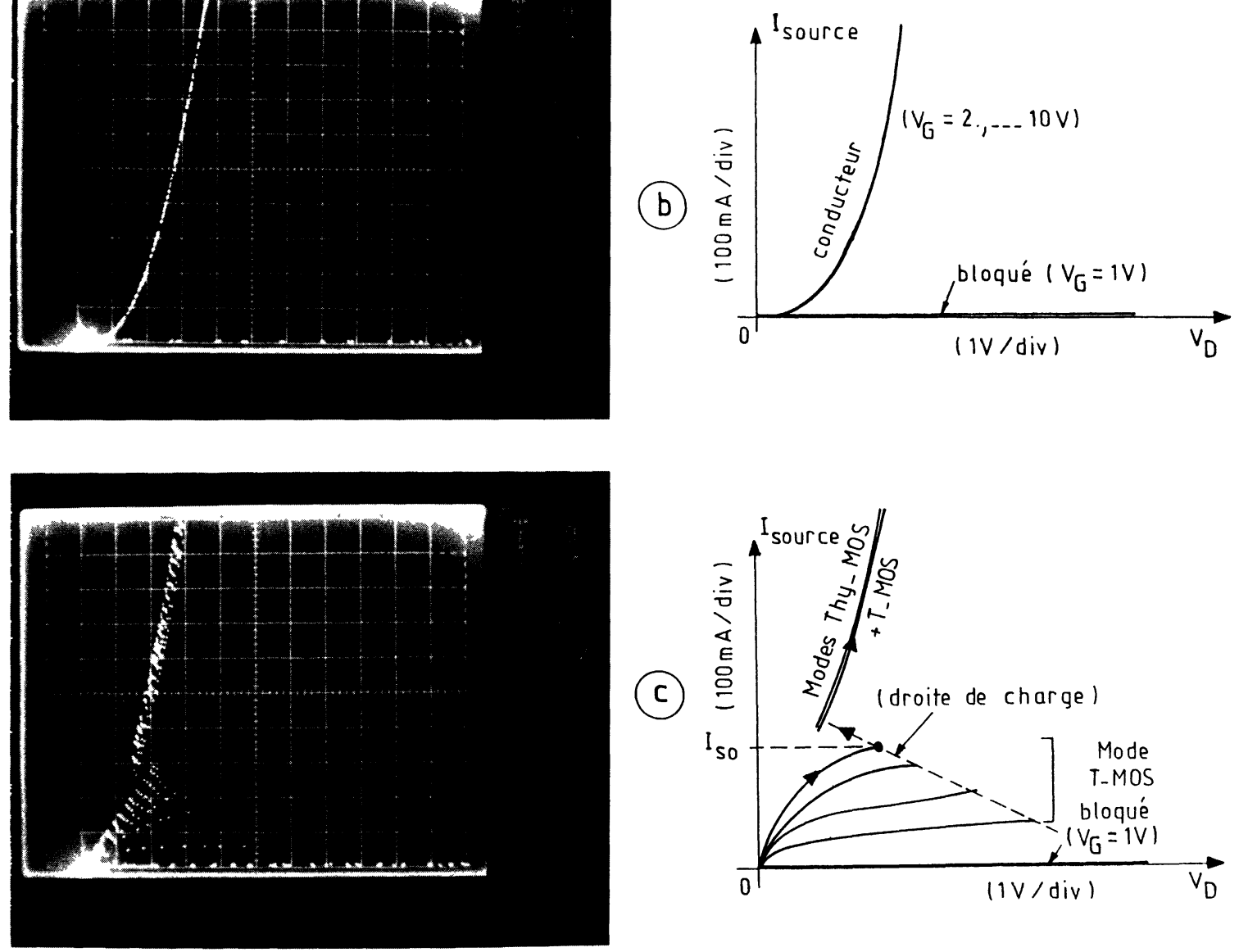

triques diverses. On peut ainsi, en mode normal, appliquer une tension positive :

i) soit sur le drain et obtenir un fonctionnement en mode transistor MOS qui présente les caractéristiques de la figure 2.1a. Dans ce cas, l'anode peut être soit laissée en l'air, soit connectée à la source. La grille constitue l'électrode de commande;
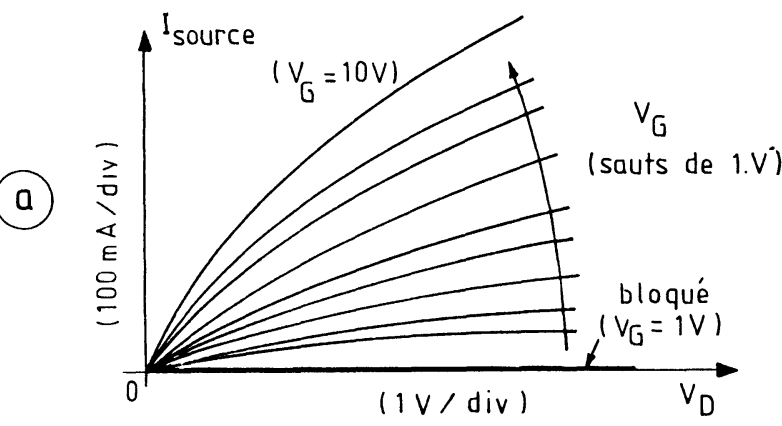

Fig. 2.1. - Caractéristique de sortie $I_{\mathrm{so}}\left(V_{\mathrm{D}}\right)$ en mode normal, dans les trois configurations : a) Mode T MOS (sortie en D). b) Mode Thy MOS (sortie en A). c) Mode $\mathrm{T}^{2}$ MOS (sortie en A + D).

[Output characteristics $I_{\text {so }}\left(V_{\mathrm{D}}\right)$ in the three operating modes : a) T MOS (output by D). b) Thy MOS (output by A). c) $\mathrm{T}^{2}$ MOS (output by D $\left.+\mathrm{A}\right)$.] 
ii) soit sur l'anode, le drain restant en l'air pour obtenir des caractéristiques de type thyristor MOS; la figure $2.1 \mathrm{~b}$ en est un exemple. On note que sur les composants que nous avons réalisés, le déclenchement entre états bloqué et passant se produit de manière très brusque juste lorsque la tension de grille atteint la tension du "seuil d'inversion" de la couche $P$. Cette sensibilité élevée à la transition entre les états bloqué et passant est liée à la valeur élevée de la résistance $R_{1}$ due principalement à notre choix de l'interdistance entre les diffusions localisées $\mathrm{P}^{+}$;

iii) soit enfin sur les électrodes de drain et d'anode reliées pour obtenir une caractéristique qui est la superposition des deux précédentes : allure de T MOS pour les très faibles tensions de drain puis caractéristique Thy MOS au-delà d'un certain seuil en courant (Fig. 2.1c).

Lorsque la tension grille-source est nulle, le composant soutient une tension bloquée voisine de 100 volts (Fig. 2.2).
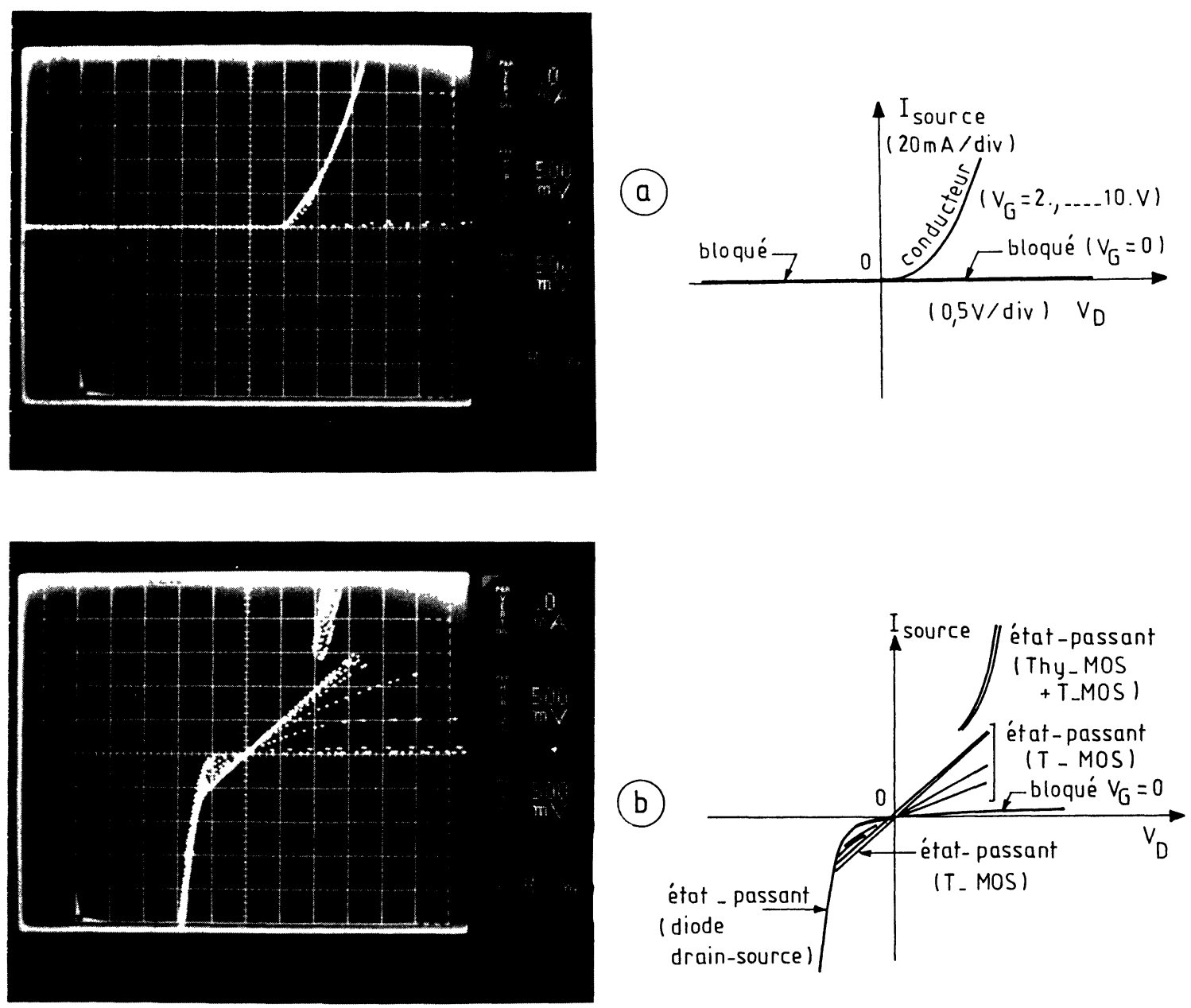

Fig. 3. - Exemple de caractéristiques courant-tension dans les quatre quadrants - le paramètre est la polarisation grillesource variant de 0 à 5 V. a) Mode Thy MOS. b) Mode T² MOS.

[Forward and reverse output characteristics in the $\mathrm{T}^{2}$ MOS operating modes - with the gate bias varying from 0 to $5 \mathrm{~V}$.] 
Les fonctionnements électriques du T MOS [10] (cas i) et du Thy MOS (cas ii) [9] ont déjà été analysés par ailleurs. En ce qui concerne le fonctionnement en mode $\mathrm{T}^{2} \mathrm{MOS}$, il apparaît nécessaire pour assurer le basculement entre les modes T MOS et Thy MOS, qu'une partie du courant d'électrons initialement horizontal - circulant entre drain et source à faible tension drain-source - soit déviée vers l'électrode d'anode. Pour ce faire, deux conditions sont à remplir :

i) la chute de tension interne créée par le courant du transistor MOS - noté $I_{\text {Mos }}$ sur la figure 1.3 à travers la résistance "drift " horizontale $R_{2}$, due à la couche épitaxiée entre canal et drain, doit être supérieure à la hauteur de barrière, $\varnothing$, de la jonction $\mathrm{N}^{-} \mathrm{P}^{+}$constituée par la couche épitaxiale $\mathrm{N}^{-}$et la couche d'anode $\mathrm{P}^{+}$;

ii) les relations de "gain interne" imposées, sur le schéma équivalent de la figure 1.3, par les gains des deux transistors bipolaires, du transistor MOS, par la résistance de " base " $R_{1}$, établies antérieurement [9, 16], doivent être respectées.

Dans nos prototypes, c'est la cơndition (i) qui est fondamentale; le critère (ii) est toujours satisfait à cause de la valeur suffisante du " gain interne", principalement lié à la valeur élevée de la résistance $R_{1}$, point qui a déjà été évoqué. De sorte que le décrochement, sur les caractéristiques de sortie, se produit pour une valeur critique du courant de source notée $I_{\mathrm{so}}\left(V_{\mathrm{G}}\right)$ définie par la condition suivante :

$$
I_{\mathrm{so}}\left(V_{\mathrm{G}}\right) \times R_{2}\left(V_{\mathrm{G}}\right)=\varnothing .
$$

En dessous de ce courant, le dispositif fonctionne en mode T MOS, au-dessus le composant est devenu un thyristor MOS. On peut noter, au passage, que la résistance $R_{2}$ est en partie modulée par la tension grillesource puisque la grille recouvre, plus ou moins selon la géométrie, la région $\mathrm{N}^{-}$" drift " et crée à sa surface une couche accumulée dont la conductivité varie [10]. Notons également que l'utilisation d'une telle structure permet, par mesure du courant de basculement $I_{\mathrm{so}}\left(V_{\mathrm{G}}\right)$ et application de la relation (1), la déterminatión originale et précise de la valeur de la résistance "drift» $R_{2}\left(V_{\mathrm{G}}\right)$ du D MOS latéral. C'est, à notre connaissance, la seule méthode proposée à ce jour qui permette cette détermination directe.

Pour être complet, nous indiquerons que les caractéristiques inverses du composant sont modifiées, lorsqu'on passe du mode Thy MOS au mode $\mathrm{T}^{2}$ MOS. La première configuration est bloquante, la deuxième analogue à celle du T MOS - est passante. La figure 3 illustre ces faits par la représentation des réseaux de caractéristiques, dans les 4 quadrants $I(V)$, relevés sur un prototype.

\section{Commentaires.}

Le $\mathrm{T}^{2}$ MOS que nous venons de présenter a une configuration technologique qui est une superposition

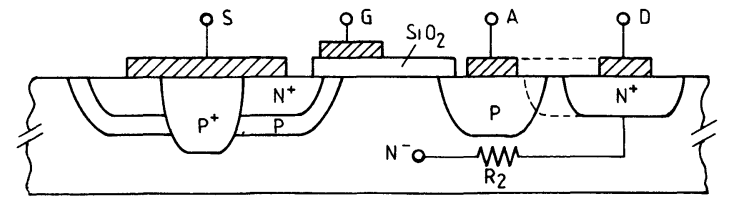

4.1

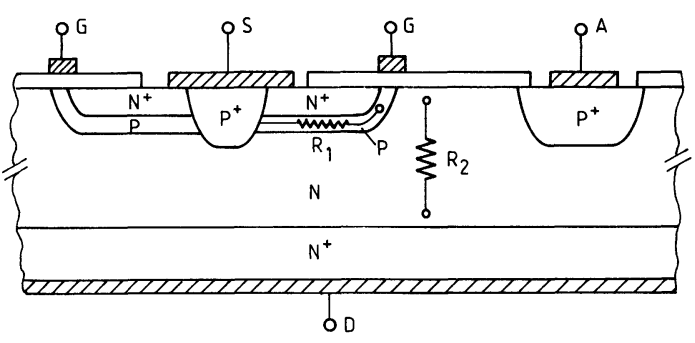

4.2

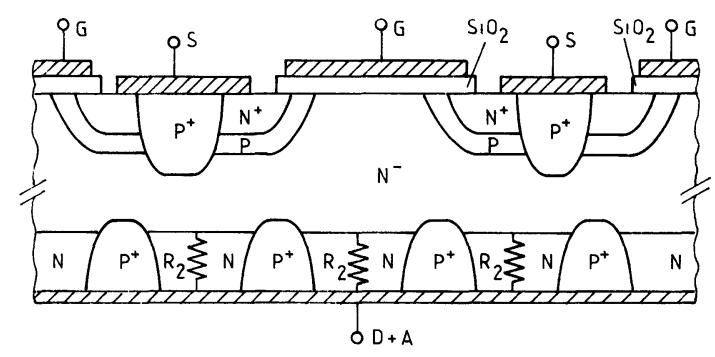

4.3

Fig. 4. - Configurations envisageables pour le $\mathrm{T}^{2} \mathrm{MOS}$ : 4.1) Configuration coplanaire. 4.2) Configuration verticale (A et D distincts). 4.3) Configuration verticale (A et D communs).

[Various geometries for the $\mathrm{T}^{2}$ MOS : 4.1) Planar. 4.2) Vertical geometry with distinctive anode $\mathbf{A}$ and drain $\mathbf{D}$ electrodes. 4.3) Vertical geometry with common $\mathrm{A}$ and $\mathrm{D}$ electrodes.]

d'un transistor MOS et d'un thyristor MOS. Il conduit en régime direct d'abord comme un D MOS, il peut ensuite basculer sur une caractéristique de type thyristor. La valeur du courant critique de basculement vient d'être précisée. En régime inverse, ce composant se comporte comme une "diode T MOS". Nous avons illustré, par une configuration particulière des électrodes, un exemple de faisabilité d'un tel dispositif. Bien entendu, tous les aspects relatifs à l'optimisation - phases technologiques plus sophistiquées, amincissement du substrat, tenue en tension, chutes de tension à l'état passant... - sont à traiter dans le futur.

Il est possible d'envisager d'autres configurations coplanaire (Fig. 4.1) ou verticales (Figs. 4.2 et 4.3) pour assurer la même fonction électrique. La première structure (4.1) est un D MOS latéral auquel a été rajouté une diffusion $P$ contiguë au drain; la résistance de basculement $R_{2}$ est localisée sous cette diffusion. La deuxième (4.2) est une configuration inversée par rapport au dispositif (1.1) : anode sur la 
face supérieure et drain sur la face inférieure. La troisième structure (4.3) est un D MOS vertical sur substrat massif ou épitaxial $\mathrm{N}^{-}$; le fond du substrat a été diffusé et se présente sous la forme de doubles motifs $\mathbf{N}$ et $\mathrm{P}^{+}$contigus et positionnés de manière quelconque vis-à-vis des éléments de la face supérieure. La résistance $R_{2}$ est localisée dans le motif $\mathrm{N}$ pincé entre deux motifs $\mathrm{P}^{+}$. Pour des nécessités de montage, drain et anode sont obligatoirement reliés dans cette dernière configuration. Il est toutefois bien évident, à ce stade d'analyse, qu'une étude plus approfondie de la conception et des propriétés électriques devient indispensable afin de pouvoir mieux cerner les qualités et défauts de ces nouveaux composants et d'en établir des règles de réalisation plus quantitatives.

Enfin, on peut évoquer quelques possibilités d'application de ce dispositif. En tant qu'élément discret, il apparaît que certains domaines d'utilisation des composants plus anciens tels que le T MOS ou le « Reverse Conducting Thyristor " $[11,12]$ pourront devenir ceux des $\mathrm{T}^{2}$ MOS; la commutation bidirectionnelle des signaux alternatifs, par utilisation en configuration série tête-bêche, sources reliées, commande entre grilles communes et sources de deux $\mathrm{T}^{2} \mathrm{MOS}$, en est un exemple d'application. La figure 5 montre les résultats obtenus en associant deux dispositifs à faible calibre en courant. La transition entre les modes $\mathrm{T}$ MOS et $\mathrm{T}^{2}$ MOS y est mise en évidence; l'avantage de la configuration $\mathrm{T}^{2}$ MOS par rapport à la configuration T MOS [13] apparaît qualitativement si on compare les chutes de tension dans ces deux modes.

Toujours en tant qu'élément discret, il est concevable de mettre à profit le principe du "saut " depuis la caractéristique T MOS sur la caractéristique Thy MOS pour envisager une "protection intégrée" dans les transistors MOS de puissance soumis à des surcharges de courant. Ceci implique bien sûr de modifier sensiblement la technologie et la structure du T MOS standard pour que celle-ci devienne celle d'un $\mathrm{T}^{2}$ MOS qui aura à fonctionner en régime normal selon le mode $\mathrm{T}$ MOS et aura à passer en régime Thy MOS sur la surcharge de courant. L'avantage majeur serait alors celui d'éviter que le TMOS atteigne lors de cette surcharge, la limite la plus critique de son aire de sécurité à savoir le lieu de passage en régime destructif de deuxième claquage [14].

En tant qu'élément intégrable, c'est-à-dire entièrement compatible avec la technologie planar associant : i) des circuits intégrés MOS ou (et) bipolaires ou (et) ii) des éléments "interrupteurs" de sortie de puissance en technologie MOS - transistors MOS, COMFET [15] ou IGT [2], thyristor MOS - l'utilisation du $\mathrm{T}^{2}$ MOS ouvre une voie nouvelle. Il s'agit de la possibilité de modifier la caractéristique ou la fonction électrique des "interrupteurs " par un seul niveau de masque, celui de gravure des interconnexions par métallisation : on obtiendra ainsi, à la demande, à partir d'un seul " élément prédiffusé » soit un T MOS, soit un Thy MOS, soit un $\mathrm{T}^{2}$ MOS.

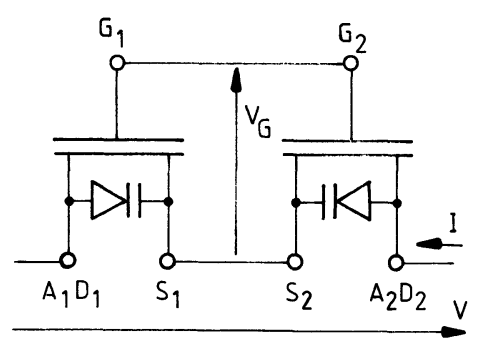

5.1

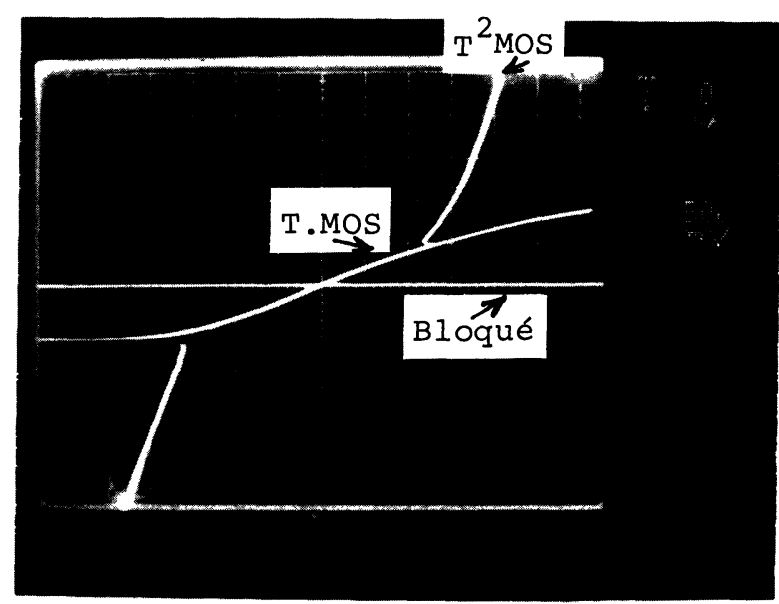

5.2

Fig. 5. - Exemple d'interrupteur bidirectionnel par association tête-bêche de deux $T^{2}$ MOS. 5.1) Configuration électrique. 5.2) Caractéristiques courant-tension : i) dans l'état bloqué $\left(V_{G}=0\right)$, ii) dans l'état conducteur T MOS $\left(V_{\mathrm{G}}=2,5 \mathrm{~V}\right)$ et iii) dans l'état conducteur $\mathrm{T}^{2} \operatorname{MOS}\left(V_{\mathrm{G}}=\right.$ $2,5 \mathrm{~V})$.

[Bidirection switch made of two " head to tail " $\mathrm{T}^{2}$ MOS. 5.1) Electrical circuit. 5.2) Output characteristics : i) in the off-state, i.e. $V_{\mathrm{G}}=0$, ii) in the T MOS on-state with $V_{\mathrm{G}}=2.5 \mathrm{~V}$, and iii) in the $\mathrm{T}^{2}$ MOS On-state with $V_{\mathrm{G}}=$ $2.5 \mathrm{~V}$.]

Ce nouveau concept qui est celui de la modification de la fonction électrique de l'élément de sortie de puissance, est l'application directe du principe mis en ouvre dans le domaine plus ancien des circuits intégrés dits prédiffusés. Il constitue, à notre avis, un pas de plus très important dans la voie récente de la conception des circuits intégrés de puissance.

\section{Remerciements.}

Nous remercions pour ses contributions et discussions le Pr. Ph. Leturcq. Les masques d'étude ont été réalisés par M. C. Solano, et les étapes du processus technologique ont été effectuées par Madame F. Rossel. Nous les remercions pour leur collaboration. 
Bibliographie

[1] Wrathall, R. S., Tam, D., Terry, L., RopP, S. P., Integrated Circuits for the Control of High Power, Proc. of the I.E.D.M., Washington (1983), p. 408411.

[2] Adler, M. S., OWyang, K. W., Baliga, B. J., Koxosa, R. A., The Evolution of Power Device Technology, IEEE Trans. Electron Devices ED 31, № 11 (1984) 1570-1591.

[3] Parker, R., Power and Logic Devices Are Merging On The Sampe Chip, Comput. Des. (1984) 29-35.

[4] Smart Power Technology Ushers in era of Logic and Power On A Single Chip Electronics 57, No 11 (1984) 87-91.

[5] Fellinger, C., Leipold, L., Experimental Study of a 1000 V SIPMOS Transistor with Integrated LowVoltage Logical Circuitry, Siemens Forsch. Entwicklungsber. 13, № 4 (1984), 166-179.

[6] Schultz, W., Smart-Power Process Puts Overvoltage Protection On Chip, Electronics 57, № 6 (1984) 134-135.

[7] Ludikhuize, A. V., High Voltage D MOS And P MOS in Analog IC's, Proc. of the I.E.D.M., San Francisco (1982), p. 81-84.

[8] Leipold, L., Baumgartner, W., Ladenhauf, W., STENGL, J. P., A FET Controlled Thyristor in SIPMOS Technology, Proc. of the I.E.D.M., Washington (1980), p. 79-82.

[9] HACHAD, S., Contribution à l'étude des mécanismes de commutation bistable dans les associations monolithiques de composants bipolaires et MOS, Thèse de 3e Cycle, Université Paul Sabatier, Toulouse, No 3029 (1984).
[10] Sanchez, J. L., Propriétés à l'état passant des transistors D MOS de puissance coplanaires ou verticaux, Thèse de $3 \mathrm{e}$ Cycle, I.N.S.A., Toulouse, № 138 (1984).

[11] Kokosa, R. A., TuFT, R. B., A High Voltage High Temperature Reverse Conducting Thyristor, IEEE Trans. Electron Devices ED 17 (1970) 667.

[12] Sze, S. M., Physics of Semiconductor Devices 2nd Ed. (John Wiley and Son) 1981, p. 222.

[13] Heinzer, W., V MOS. A solution to High Speed, High Current, Low Resistance Analog Switches, " Application Note Siliconix », AN 77-2 (1977).

[14] Tranduc, H., Rossel, P., Sanchez, J. L., Premier et second claquages dans les transistors MOS, Revue Phys. Appl. 19, № 10 (1984) 859-878.

[15] Russel, J. P., Goodman, A. M., Goodman, L. A., Neilson, J. M., The COMFET. A New High Conductance MOS. Gated Device, IEEE Electron Device Lett. ED L 4, № 3 (1983) 63-65.

[16] Hachad, S., Cros, C., Darees, D., Dorkel, J. M., LETURCQ, P., Latchup Criteria in Insulated Gate PNPN Structures, IEEE Trans. Electron Devices ED 32, No 3 (1985) 594-598.

[17] Hu, C., Chi, M. H., Patel, V. M., Optimum Design of Power MOSFET's, IEEE Trans. Electron Devices ED 31, No 12 (1985) 1693-1700. 